\title{
Ökonomisierung der Medienindustrie aus wirtschaftspolitischer Perspektive
}

\author{
Andrea Grisold
}

Eine Betrachtung der Ökonomisierung der Medien aus wirtschaftspolitischer Perspektive führt zum in seiner wechselseitigen Bedingtheit komplexen und widersprüchlichen Verhältnis von Ökonomisierung und Wirtschaftspolitik: Viele wirtschaftspolitische Maßnabmen beschleunigen die Ökonomisierung des Mediensektors, andererseits verlangt eine größere Ökonomisierung - die mit erhöhter Marktausrichtung auch zu gesellschaftlich unerwünschten Ergebnissen fübren kann - nach erböbten (wenngleich anders ausgerichteten) wirtschaftspolitischen Eingriffen. Dieser Artikel stellt die unterschiedlichen ökonomischen Zugänge zu Wirtschaftspolitik und deren Ausrichtung dar, gibt eine Darstellung derjenigen wirtschaftspolitischen Aktivitäten, die der Ökonomisierung förderlich waren oder sind, und schließt mit einem Katalog an wirtschaftspolitischen Instrumenten, die als countervailing power zu den negativen Effekten einer verstärkten Ökonomisierung aus ökonomischer Perspektive heraus wünschenswert und sinnvoll wären.

Analysegegenstand dieses Artikels ist das Verhältnis zwischen Wirtschaftspolitik und Ökonomisierung am Mediensektor. Dieses Verhältnis stellt, soweit sei die Konklusio gleich vorweggenommen, kein monokausales, sondern durchaus ein in sich widersprüchliches dar: Wirtschaftspolitische Maßnahmen sind es, die die Ökonomisierung des Mediensektors durchaus befördern, andererseits verlangt eine größere Ökonomisierung nach erhöhten (wenngleich auch anders gelagerten) wirtschaftspolitischen Eingriffen. Ich werde das Thema der Ökonomisierung der Medienindustrie aus wirtschaftspolitischer Perspektive im Folgenden mit einer Begriffsklärung von Wirtschaftspolitik und Ökonomisierung beginnen, mit einem theoretisch-ökonomischen Diskurs zu Notwendigkeit und Aufgaben von Wirtschaftspolitik fortführen, und danach auf das - zumindest auf den ersten Blick - widersprüchliche Verhältnis von Wirtschaftspolitik und Ökonomisierung des Mediensektors eingehen.

\section{Wirtschaftspolitische Grundlagen: Ziele, Funktionen und Instrumente}

In einer ersten Definition kann Wirtschaftspolitik als „Durchführung von Maßnahmen, mit denen bestimmte ökonomische und soziale Ziele verfolgt werden sollen“" (Altmann $2000,4)$ bezeichnet werden. Dies wird in einer grundsätzlich marktwirtschaftlich ausgerichteten ökonomischen Ordnung dann notwendig, wenn der privatwirtschaftliche Prozess nicht die gewünschten Resultate erbringt, also etwa bei wirtschaftlichen Instabilitäten wie Rezessionen, Arbeitslosigkeit, Inflation. Politische Eingriffe können sich auch aus sozialpolitischen Umverteilungsbestrebungen des Ergebnisses von Marktprozessen ergeben oder daraus, dass das Ausschlussprinzip der Nutzung von spezifischen Gütern nicht erwünscht ist. Weiter können auch negative externe Effekte (privates Handeln verursacht Kosten, die von der Allgemeinheit getragen werden müssen) wirtschaftspolitische Maßnahmen erforderlich machen.

Als oberstes Ziel einer Theorie der Wirtschaftspolitik wird „gesellschaftliche Wohlfahrt“ definiert (z. B. in Berg/Cassel 1995 oder Nowotny 1997). Wenn diese „gesellschaftliche Wohlfahrt“ von kritischen Stimmen gleichermaßen zu Recht wie zu Unrecht 
als Leerformel bezeichnet wurde, so will das kurz erläutert werden: Wirtschaftspolitik bedient sich immer auch idealistischer Zielsetzungen. Selbst wenn diese aufgrund starker Interessensdominanzen nicht in eine entsprechende Praxis umzusetzen sind, so darf doch auf diese Zielsetzungen nicht verzichtet werden im Sinne von Leitwerten, an denen es das Ergebnis von wirtschaftspolitischer Arbeit zu messen gilt.

Im so genannten „Magischen Vieleck“ werden die wirtschaftspolitischen Ziele folgendermaßen aufgelistet: Stabilität des Preisniveaus, hoher Beschäftigungsstand, außenwirtschaftliches Gleichgewicht und hohes Wirtschaftswachstum als Hauptziele; weitere Ziele sind Verteilungsgerechtigkeit, Umweltschutz, etc. Die Politikfelder und Instrumente, die damit einhergehen, sind vielgestaltig und werden an dieser Stelle nur beispielhaft angeführt, nicht vollständig aufgezählt: So unterschiedliche Bereiche wie Fiskalpolitik, Wettbewerbspolitik, Agrarpolitik, Geld- und Währungspolitik, Strukturpolitik etc. zählen dazu.

Für die hier betrachtete Medienwirtschaftspolitik sind vor allem die Ziele Wirtschaftswachstum, hoher Beschäftigungsstand und Verteilungsgerechtigkeit relevant, wie sich zum anderen auch die Bereitstellung öffentlicher Güter, hoher Konzentrationsgrad bei Medienunternehmen, externe Effekte wie generell Informationsasymmetrien als Gründe für Medienwirtschaftspolitik anführen lassen.

Der Rolle der Wirtschaftspolitik kommt je nach volkswirtschaftlicher Schule unterschiedliche Funktion zu. Zwei Prototypen ökonomischer Schulen lassen sich unterscheiden (Rothschild 1996): Die neoklassische und die keynesianische Sichtweise. Ergänzt werden kann dies noch durch die regulationstheoretische Sichtweise, die Ökonomien immer als ein Set an unterschiedlichen regulatorischen Maßnahmen begreift, von denen Wirtschaftspolitik einen Maßnahmenkatalog darstellt, welcher aktiv ins Wirtschaftsgeschehen eingreifen kann ${ }^{1}$.

Die Neoklassik schreibt alleine dem Markt als Allokationsmechanismus Effizienz zu, und begründet dies mit den Defizienzen jeglicher anderer denn Marktregulierungsformen. Der neoklassische Zugang sieht grundsätzlich mit einer Ökonomisierung alle regulatorischen Erfordernisse erfüllt, denn endlich können sich die - selbstverständlich positiven - Marktkräfte frei entfalten und über Konsumentensouveränität die Wünsche der Nachfrager befriedigen. Doch sind auch im neoklassischen Denkgebäude unerwünschte Wirkungen des Marktes nicht gänzlich ausgeschlossen („Marktversagen“), der Wirtschaftspolitik kommt dann die Funktion eines Gegengewichtes zu, welches die negativen Effekte der rein ökonomischen Behandlung gesellschaftlicher Güter auszugleichen hat.

Eine grundlegend andere Funktion messen die in einer postkeynesianischen Tradition stehenden VertreterInnen der Politischen Ökonomie der Wirtschaftspolitik bei, für die öffentliche Institutionen aus mehreren Gründen von zentraler Bedeutung sind (Arestis/Sawyer 1998, 8): Erstens ist der öffentliche Sektor als Träger von Wirtschaftspolitik traditionellerweise Bereitsteller wichtiger Dienstleistungen wie Bildung oder

1 Die anderen Sets neben den Formen der Intervention des Staates in das Marktgeschehen sind das Geld- und Kapitalverhältnis (wie die Klärung gesellschaftlicher Besitzverhältnisse, die Strukturen des Finanzmarktes, der Horizont der Kapitalverwertung ...), das Lohnverhältnis (Form der Arbeitsorganisation und gesellschaftlicher Arbeitsteilung, Arbeitsplatzsicherheit und Mobilität, Formen der Lohnbestimmung), das Konkurrenzverhältnis der einzelnen Kapitalteile (Markt- und Betriebsstrukturen, Formen der Marktregulierung) und das Verhältnis zwischen nationalem und internationalem Regime (Boyer 1986). 
Gesundheit, Infrastruktur oder Zentralbanken, die den Finanzsektor stabilisieren; zweitens wird dem öffentlichen Sektor eine zentrale Rolle darin zugeschrieben, privatwirtschaftlichen Institutionen ihre Rahmenbedingungen aufzubereiten. Im Gegensatz zur Neoklassik werden rechtliche Rahmenbedingungen alleine als nicht ausreichend erachtet, sondern es bedarf einer umfassenden Palette an Regulierungsaktivitäten. Ein wesentlicher Unterschied zwischen der Neoklassik und der Politischen Ökonomie manifestiert sich also darin, welche Rolle dem Markt zugeschrieben wird, und welche Wichtigkeit demgegenüber der Wirtschaftspolitik zukommt. Wenn der Markt nur im Ausnahmefall zu produktiver und allokativer Effizienz führt, wenn ein allgemeines Gleichgewicht nicht herstellbar ist ${ }^{2}$, dann kommt der Wirtschaftspolitik eine elementar wichtigere Rolle zu als im neoklassischen Denkgebäude (Rothschild 1996, 45f). ${ }^{3}$

Während eine keynesianische Wirtschaftspolitik Regulierungen aufgrund von umfassendem Marktversagen begründete und entsprechende marktregulierende Eingriffe vornahm, ist die neoliberale Regulierungsdiskussion auf die Beseitigung von Staatsversagen konzentriert. Ausgangspunkt neoklassischer Regulierungstheorien ist, dass staatliche Eingriffe zeitlich unexakt, nicht effizient und damit gegenstandslos bis kontraproduktiv wirken. Von regulierungstheoretischer Seite wird auch oft argumentiert, dass Regulierungen vorrangig bis ausschließlich großen Lobbying-Gruppen zu Gute kommen ${ }^{4}$. Die an die neoklassische Tradition anknüpfenden Regulierungstheorien werden üblicherweise in normative und positive unterteilt. Während normative Regulierungstheorien darstellen, wann reguliert werden soll, gehen positive von der Fragestellung aus, wer, wann und wie reguliert; Regulierer agieren im zweiten Fall sicher nicht neutral, sondern interessengeleitet. Nun ist die Annahme, die Regulierten würden ihre Regulierungsformen mitbestimmen, keineswegs falsch. Die Schlussfolgerung, gerade aus diesem Grund den Markt als neutrale Instanz wirken zu lassen, stellt aber ein zumindest ebenso utopisches Konzept dar; nicht auf Grund mangelnder Durchsetzbarkeit auf der politischen Ebene, sondern weil die idealen Marktbedingungen, die dafür notwendig wären, nicht vorzufinden und auch nicht erzeugbar sind. Sollen Regulierungsmaßnahmen festgelegt werden, ist von Verhandlungen und einem Tauziehen verschiedener Interessengruppen auszugehen. Dabei stellt sich das Problem, welche Gruppen über adäquate Artikulationsweisen und Durchsetzungsmacht verfügen und welche nicht. Dies ist der Ausgangspunkt von theoretischen Ansätzen, die Regulierung nicht mehr als Sonderfall und nicht mehr als dem Funktionieren des ökonomischen Prozesses hinderlich darstellen.

Wenn das Nichtzutreffen neoklassischer Annahmen nun nicht mehr die Ausnahme darstellt, wie dies im Falle von „Marktversagen“ bereits im Terminus anklingt, sondern - wie etwa in der keynesianischen Theorie oder der französischen Regulationstheorie als signifikantes Bestimmungsmerkmal des ökonomischen Systems selbst identifiziert wird, so sind Regulierungsaktivitäten ebenso nicht mehr nur in vielen Ausnahmefällen notwendig, sondern müssen als wesentliches Moment für das effiziente Funktionieren unserer kapitalistisch strukturierten Ökonomie angesehen werden.

2 ... die Suche danach daher aussichtslos ...

3 Für eine Zusammenfassung unterschiedlicher Regulierungstheorien in Bezug auf die Medienindustrie siehe Grisold (1996 oder 1998).

4 Siehe dazu an Standardwerken: Stigler (1971) oder Peltzman (1976); als Überblick an aktueller Literatur: Stigler (1995). 


\section{Begriff und Ursachen der Ökonomisierung}

Der Begriff der Ökonomisierung kann von unterschiedlichen Seiten her gesehen werden: Über Individuen betrachtet, versteht man darunter eine stärkere Nutzenorientierung und individuelle Nutzenmaximierung; produktionsorientiert wird darunter der optimale Einsatz von Ressourcen verstanden; und als Politikorientierung heißt es ein verstärktes Vertrauen auf Marktkräfte und die positiven Auswirkungen des Wettbewerbs. Die Herausbildung individueller Verhaltensweisen ausklammernd ${ }^{5}$ kann für eine wirtschaftspolitische Perspektive der Begriff der Ökonomisierung wie folgt aufgeschlüsselt werden:

1. Wie Güter und Dienstleistungen bei gegebenen Inputfaktoren am besten produziert werden, ohne Ressourcen zu vergeuden. Dies ist eine sehr neutrale Definition von Ökonomie, Ökonomisierung könnte als Weg zu diesem Idealzustand beschrieben werden. ${ }^{6}$

2. Ein verstärktes Rekurrieren auf den „Markt“ als bestes Allokationssystem: Unternehmensziele wandeln sich zur vorrangigen Profitorientierung (dies wird in der Medienwissenschaft oft auch als „Kommerzialisierung“ bezeichnet), Marktbeziehungen weisen eine stärkere Wettbewerbsorientierung auf.7 Wenn der Wandel der Unternehmensziele aufgrund der Änderungen von öffentlichen zu privatwirtschaftlichen Eigentumsverhältnissen bedingt wurde, so war damit immer eine wirtschaftspolitische Entscheidung verknüpft. Ob Medienprodukte über ein marktliches Instrumentarium am besten bereitgestellt werden können, wird im Folgenden zu diskutieren sein. Die Veränderung der Marktsituation auf gesamtgesellschaftlichem Niveau wird durch Wirtschaftspolitik zumindest begleitet, wenn nicht geleitet. ${ }^{8}$

Eine verstärkte Marktorientierung und damit Profitorientierung ist als positiv einzustufen, wenn über erhöhten Effizienzdruck Kostensenkungen erreicht und diese auch auf die Preise überwälzt werden. Sie ist dann als negativ anzusehen, wenn der Markt als unvollkommener dazu führt, dass Produkte, für die es eine Nachfrage gäbe, nicht angeboten werden bzw. nur zu überhöhten Preisen, ebenso dann, wenn der Markt zu Unternehmenskonzentration führt und dazu, dass gesellschaftliche Funktionen von Gütern und Dienstleistungen vernachlässigt werden.

Dies vorausgesetzt, wird die verstärkte Ausrichtung auf den Markt als bestes Allokationssystem, verbunden mit erhöhter Profitorientierung, Einfluss der Werbewirtschaft und dem Bemühen um Effizienzsteigerung im Folgenden als Ökonomisierung bezeichnet. Im Rundfunksektor kann Ökonomisierung im Sinne einer zunehmenden Dominanz der über den Markt angebotenen Rundfunkprodukte als Folge von Privatisierungen verstanden werden. Da sich die Ursachen der Ökonomisierung für die unterschiedlichen Medienbranchen durchaus unterschiedlich darstellen, möchte ich an dieser Stelle

5 Siehe dazu z. B. Pirker (1999), wie auch den Artikel von Heinrich in diesem Band.

6 Wobei diese Handlungsanleitung in der Praxis so eindeutig nicht ist: Wer definiert, welche Inputfaktoren das „beste“ Ergebnis bringen? So ist das Billigste nicht immer auch mit dem Besten gleichzusetzen, oder, anders ausgedrückt, stellen z. B. Kreativität und Effizienz zwei widerstrebende Zielkriterien dar.

7 Welcher dieser beiden Faktoren zuerst initiierend den anderen bedingte, muss an dieser Stelle unbeantwortet bleiben.

8 Über den Abbau von Handelshemmnissen, über vorrangige Wettbewerbsförderung, über fehlende flankierende Maßnahmen zur sozialen Verträglichkeit etc. 
den Rundfunksektor als Beispiel heranziehen, um aus wirtschaftspolitischer Perspektive die Ökonomisierungsursachen darzustellen: ${ }^{9}$

- Politikausrichtung: Zunehmende Wirkungsmächtigkeit neoliberaler Politik, besonders auch innerhalb der Europäischen Union, wie dies im Katalog der vier Grundfreiheiten des Binnenmarktes (freier Verkehr von Waren, Personen, Dienstleistungen und Kapital) bereits formuliert ist.

- Ausrichtung der Wirtschaftspolitik: Über eine Verschiebung des Problembewusstseins von Marktversagen hin zu Staatsversagen ergab sich eine Auffassungsänderung über Rolle, Funktion und Gestaltung von Wirtschaftspolitik. ${ }^{10}$

- Marktveränderungen über Gewinnerwartungen: Rundfunk wurde, über eine Erhöhung der gesamtwirtschaftlichen Werbeaufwendungen, als profitabler Wirtschaftszweig erkannt; die Einführung privatwirtschaftlicher Unternehmen führte zu neuen Marktverhältnissen.

- Entwicklung am Technologiesektor: Neue Technologien ermöglichten die vermehrte Einrichtung neuer Rundfunkstationen: Mehr Frequenzen stehen zur Verfügung, Kabel und Satelliten-Verteilsysteme, gekoppelt mit der Entwicklung im Telekommunikationsbereich, führen zu effizienterer Übertragungstechnik: Dies galt noch vor der Einführung der Digitalisierung bereits für die Breitband-Technologie.

- Soziale Bewegungen als neue politische Größe: Die politische und kulturelle Aufbruchstimmung der späten 60er Jahre markierte den Beginn der Ära der „Liberalisierung“ des Rundfunks in Österreich: Neue soziale Bewegungen gründeten nichtkommerzielle Stationen als Gegenbewegung zu staatlicher Dominanz (speziell im Radiobereich). Wenn dies auch keineswegs aus ökonomischen Gründen geschah, so trug es dennoch in der Folge zur Ökonomisierung bei, da sich die Stationen aus betriebswirtschaftlichem Kalkül bald zusammenschlossen, zu ihrer Finanzierung auf Werbung nicht verzichten wollten oder konnten und so - indirekt und unintendiert - die Ökonomisierung des Rundfunksektors beförderten. ${ }^{11}$

Für die Betrachtung von Ökonomisierung aus wirtschaftspolitischer Perspektive stellt sich somit erstens die Frage, wie über Wirtschaftspolitik die Ökonomisierung am Mediensektor konkret befördert wurde und wird, sowie zweitens, wie die zu beobachtenden negativen Auswirkungen der Ökonomisierung - wenn marktliche Bereitstellung der Mediengüter nicht zu optimalen Ergebnissen führt - am Mediensektor durch wirtschaftspolitische Instrumente zu verringern wären.

\section{3. Ökonomisierung der Medienbranche durch Wirtschaftspolitik}

Jenes Verständnis von Ökonomisierung, das dem Markt vorrangige Gestaltungskompetenz zuweist, hat seinen Niederschlag nicht zuletzt auch im wirtschaftspolitischen Bereich gefunden: Erstens theoretisch im Sinne einer Beschränkung von Wirtschaftspolitik auf Wettbewerbspolitik, welche sich in der Praxis zumeist aber als Ermöglichung großer Unternehmenseinheiten darstellt. So ist eine reduzierte Rolle von Wirtschaftspolitik als gesellschaftlich gestalterisches Element auf die Etablierung funktionierender

9 Ich stütze mich dabei auf Collins/Garnham/Locksey (1988), McQuail (1998), und Altvater (1997).

10 Siehe dazu detaillierter: Kapitel 3 und 4.

11 Dieser Punkt wurde angeführt, um auch ein Beispiel für indirekte Ökonomisierung aufzuzeigen. 
Unternehmenseinheiten am Beispiel des österreichischen Privatradiogesetzes ohne Schwierigkeiten nachzuweisen (Götschl 1998). Zweitens fand und findet unter den so geschaffenen veränderten Rahmenbedingungen eine Verschiebung der Eigentumsstrukturen im Rundfunk zugunsten vermehrt privatwirtschaftlich geführter Unternehmen statt. Drittens schließlich ist ein Abrücken der Wirtschaftspolitik von der Überzeugung, Massenmedienprodukte seien als öffentliche bzw. meritorische Güter zu behandeln, festzustellen ${ }^{12}$. Damit verbunden ist, dass das Problem des öffentlichen Interesses an diesen Produkten von Seiten der Wirtschaftspolitik nur mehr nachrangig behandelt wird.

Da Wirtschaftspolitik immer auch Interessenpolitik darstellt, wirft sich die Frage auf, wie solche Interessen vertreten werden können, die weder in Kaufkraft noch in politischem Lobbying zum Ausdruck kommen. ${ }^{13}$ Massenmedien schreiben sich selbst gerne die Funktion einer „countervailing power“ (Gegenmacht) zu, die sie, wenn überhaupt, am nachdrücklichsten gegenüber der Politik einsetzen. Sind ökonomische Interessen respektive Interessensgruppen angesprochen, treten Medien hingegen viel weniger deutlich in Erscheinung (z. B. in unternehmenskritischer Berichterstattung ${ }^{14}$ ).

Welche wirtschaftspolitischen Instrumente werden nun im Sinne der Beförderung einer Ökonomisierung am Mediensektor eingesetzt? Im Folgenden soll anhand von unterschiedlichen wirtschaftpolitischen Instrumenten am Beispiel des audiovisuellen Sektors die Problematik dargestellt werden.

Derzeit reduziert sich Wirtschaftspolitik oftmals auf Wettbewerbspolitik, mit dem als Ideal formulierten Ziel, eine Vielzahl von kleinen, nicht marktmächtigen Unternehmen am Markt operieren zu haben. Kann dieser Idealzustand hergestellt werden, so sorgen die positiven Kräfte des Wettbewerbs für ein reibungsloses Funktionieren des Marktes. Aufgrund der Kostenstrukturen und spezifischen Gütereigenschaften ist diese Wettbewerbsform allerdings am Mediensektor ökonomisch oft ineffizient bzw. nicht zu erreichen, die vorrangige Ausrichtung der Wirtschaftspolitik auf Wettbewerbspolitik daher wenig sinnvoll.

Realiter wird unter dem Terminus Wettbewerbspolitik oft Standortpolitik betrieben (besonders für einen Wirtschaftssektor wie etwa den der elektronischen Medien, der

12 Kiefer (1997) nennt dies „Entmeritorisierung“ von Medienleistungen: ein Zurückdrängen der gesellschaftlich erwünschten meritorischen Güter zu Gunsten der auf Märkten verkäuflichen, wirtschaftlich rentablen Angebote.

13 Wobei ich hier annehme, dass Massenmedien sich um alle kaufkräftigen Schichten kümmern, was sehr wohl auch in Frage gestellt werden kann. Peterson (1982) tut das im „Production of Culture"-Ansatz, der Produktionsbedingungen in den Kulturindustrien als angebotsdeterminiert sieht; Brown $(1996,8)$ spricht von Minoritäten, welche für die Befriedigung ihrer Bedürfnisse zwar eine hohe Zahlungsbereitschaft hätten, diese aber nicht adäquat kommunizieren können. Wenn in mittlerem Alter stehende kaufkräftige Mittelschichten z. B. am Radiosektor so gar nicht bedient werden (nicht nur in Österreich, sondern auch z. B. in Irland oder in weiten Teilen Großbritanniens), weil sie entweder nicht als Zielgruppe definiert werden oder andere Werbeträger als adäquater für die zu bewerbenden Produkte angesehen werden, so kann das in einer weiteren Auslegung des Wortes als Marktversagen gesehen werden.

14 So ist z. B. in Zeitungen der Politikteil auch moralisch-kritisch gegenüber ebendieser Politik, von der berichtet wird, ausgerichtet, wohingegen der Wirtschaftsteil derselben Zeitungen sehr wenig wirtschaftskritisch in Erscheinung tritt. Dies ist über Interessen leicht erklärbar: Der Politikteil wird nicht vorrangig für Politiker geschrieben, der Wirtschaftsteil dient aber hauptsächlich - zumindest im Selbstverständnis der Zeitungen - der Information für Aktionäre bzw. Wirtschaftstreibende. 
derzeit als Wachstumsbranche schlechthin dargestellt wird). Diese Standortpolitik erfolgt aufgrund von regionalem Wettbewerb. Da der Medienbereich als Zukunftsbranche gehandelt wird, bemühen sich die Regionen verstärkt darum, Unternehmen aus dieser Branche anzusiedeln. Mittels Subventionen werden die Kosten der Unternehmen der Massenmedienindustrien verringert, über Multiplikatorwirkungen weitere Wachstumsund Beschäftigungseffekte zu erreichen gesucht.

Der Dynamik unserer Wirtschaftsform immanent ist die Bildung unternehmerischer Konzentrationsformen. Kartellrechtliche Regelungen als Teil der Wettbewerbspolitik sollen derlei negative Machtkonzentrationen nach Möglichkeit hintanhalten. Ihre Möglichkeiten sind jedoch klar limitiert: Unmittelbare wirtschaftliche Interessen nicht nur der betroffenen Unternehmen, sondern auch der Region, der Nation im Wettbewerb mit anderen Regionen respektive Nationen werden einer strikten Auslegung von Anti-TrustRegelungen immer entgegenstehen. Damit fallen kartellrechtliche Regelungen üblicherweise unter den Oberbegriff „Kosmetik“ und werden nur in den seltensten Fällen auch exekutiert ${ }^{15}$. Auf EU-Ebene stellt das Verbot des Zusammenschlusses von Bertelsmann, Kirch und Deutscher Telekom für den Pay-TV-Sektor im Mai 1998 eine der wenigen Ausnahmen dar. Im Gegensatz dazu wurde z. B. die Beteiligung von Murdochs BSkyB an KirchPayTV nicht untersagt. ${ }^{16}$ Auf nationaler Ebene sind die in Österreich geplanten Veränderungen im Regionalradiogesetz ein Beispiel für die Lockerung kartellrechtlicher Maßnahmen: Nicht nur von Regierungsseite wird eine Aufhebung der Eigentumsbeschränkungen geplant, auch die Opposition hat dagegen nichts einzuwenden ${ }^{17}$.

Wenn Massenmedienprodukte öffentliche Güter ${ }^{18}$ darstellen, und als Ziel definiert ist, die Inhalte von Massenmedien pluralistisch zu produzieren, so sind dafür Eigentumsrechte von zentraler Bedeutung. Sie sichern den Zugriff auf Produktion und Verteilung von Inhalten: Eigentumsrechte an den Distributionskanälen auf der einen, an den potenziellen Inhalten der Massenmedien auf der anderen Seite. Bei öffentlichen Gütern stellt sich die Frage, ob sie öffentlich produziert werden sollen oder privatwirtschaftlich, dann aber unter öffentlicher Kontrolle. Privatwirtschaftlich organisierte Unternehmen haben spezifische Interessenlagen, die über private Massenmedien verstärkt transportiert werden, die Generierung von Shareholder-Value steht pluralistischer Kommunikation entgegen. Eine wirtschaftspolitische Ausrichtung, die den Anteil der privatwirtschaftlichen TV-Stationen erhöht und eine Konzentration darin nicht verhindert, wird zur Folge haben, dass nicht Gewinn bringend absetzbare Inhalte nicht mehr produziert und gesendet werden.

Fiskalpolitik als Einnahmen- und Ausgabenpolitik staatlicher Bürokratien beinhaltet die Erhebung von Steuern und Abgaben ebenso wie die Vergabe von Subventionen. Förderungen können unterteilt werden in allgemeine, generell der Branche zugute kommende, und solche mit explizit normativen wirtschaftspolitischen Zielsetzungen. Sind

15 Bagdikian (1997) oder Herman/McChesney (1997) bringen eine Fülle an Beispielen für den USamerikanischen Raum.

16 Für eine genaue Begründung siehe KEG (2000).

17 Derzeit bestehende Einschränkungen des Crossownerships von marktbeherrschenden Zeitungen und Radiounternehmen in einer Region werden ebenso fallen wie Beschränkungen der Eigentumskonzentration am kommerziellen Radiosektor generell.

18 Öffentliche Güter werden über das Nicht-Ausschlussprinizip vom Konsum und über die Nicht-Rivalität im Konsum definiert; aus beiden Gründen ist eine direkte, eindeutig zuordenbare Bepreisung dieser Güter nicht möglich. 
zu zweiteren die Regelungen öffentlich-rechtlicher Rundfunkunternehmen zu zählen, haben branchenfördernde finanzpolitische Maßnahmen (wie ein geringerer Mehrwertsteuersatz, geringere Postgebühren beim Versand für den Pressesektor etc.) höhere Förderungswirkung bei großen, marktbeherrschenden Unternehmen der Medienindustrie.

\section{Wirtschaftspolitik als Folge der Ökonomisierung der Medienbranche}

Die Folgewirkungen des Prozesses der Ökonomisierung sind kaum mehr reversibel: Massenmedienprodukte werden nach rein ökonomischen Profiterfordernissen ausgerichtet, und über die Entwicklung des Marktes betrifft dies auch öffentliche Unternehmen. Dies sei am Beispiel der catch-22-Situation für öffentlich-rechtlichen Rundfunk durch die „Liberalisierung“ erläutert: Die einfachste Möglichkeit, konkurrieren zu können, ist die, gleichsam selbst zur kommerziellen Station zu mutieren (eine Strategie, die von den meisten öffentlich-rechtlichen Rundfunkstationen auch verfolgt zu werden scheint, siehe Blumler 1992, Achille/Miege 1994, Burgelmann 1997). Damit verlieren sie aber ihre Legitimation für den Bezug von Rundfunkgebühren („Wieso für etwas zahlen, das man umsonst von Privaten kriegen kann?"). Zugleich aber verlieren sie diese Legitimation auch dann, wenn sie ein Programm anbieten, das keine großen Zuseherzahlen anzieht; wobei die Argumentation dann in die Richtung geht, dass solche Programme eben nicht gewünscht würden.

Der Nobelpreisträger Ronald Coase hat bereits in den 60er Jahren in seiner Einschätzung von werbefinanzierten Rundfunkprogrammen eine einfache ökonomische Realität dargelegt: „With commercial broadcasting, the person who pays for the broadcast of a program is the advertiser. It follows that the programs broadcast are those which maximise the profits to be derived from broadcasting" (Coase 1966, 446). Der Chicago-Ökonom schlägt als Gegenmaßnahme die Entfaltung des Marktmechanismus schlechthin vor: Die Einführung von Pay-per-View, und damit die Ermöglichung des Wirkens des Preismechanismus, um Angebot und Nachfrage ins Gleichgewicht zu bringen und so Konsumentensouveränität herzustellen. Wenn allerdings nicht von derart idealistischen Annahmen bezüglich der Verteilungsgerechtigkeit ausgegangen wird, sondern von sozialer Hierarchisierung, unterschiedlichen Einkommens- und Bildungsniveaus, von Informationsasymmetrien etc., so wird eine aktive und gestaltende Wirtschaftspolitik als Regulativ notwendig.

Damit stellt sich die Frage, welche Aufgaben von wirtschaftspolitischer Seite am Mediensektor vorrangig zu übernehmen wären im Sinne ökonomischer und sozialer Ziele, wenn in einer grundsätzlich marktwirtschaftlich ausgerichteten ökonomischen Ordnung der privatwirtschaftliche Prozess nicht die gewünschten Resultate erbringt. Allgemein formuliert sind das ein freier Zugang zu Information, ein Ausgleich für die zunehmende Privatisierung öffentlicher Güter und ein Gegengewicht zur Standardisierung der Inhalte:

- Infrastrukturmaßnahmen: Wirtschaftspolitik gegenüber Massenmedien soll sicherstellen, dass ein universeller Zugang zu Massenmedienprodukten ermöglicht wird. Solch eine Infrastrukturmaßnahme kann sich nicht an marktwirtschaftlichen Kriterien orientieren.

- Wirtschaftspolitische Maßnahmen am Massenmediensektor haben sich auf die Inhalte der Massenmedien zu richten, d. h. den symbolischen Gehalt ebendieser Produkte in Rechnung zu stellen. So müsste, um eine funktionierende Gegenmacht der Massenmedien zu gewährleisten, Wirtschaftspolitik, mit Kulturpolitik gekoppelt, eine Diversifizierung der Inhalte anstreben. 
- Die Preise für solche Güter festzulegen, deren Bepreisung über den Markt nicht möglich ist, ist eine weitere Aufgabe der Wirtschaftspolitik. Preispolitik als fiskalische wirtschaftspolitische Maßnahme ist im audiovisuellen Bereich auf die Gebührenordnung für öffentlichen Rundfunk wie auch indirekt auf die Regulierung der Werbezeiten beschränkt. Auf der Gebührenseite wären aus effizienztheoretischer ökonomischer Perspektive Steuereinnahmen ${ }^{19}$ den derzeit vorherrschenden Gebührensystemen zumindest nicht unterlegen. Für eine Bereitstellung von öffentlichen Gütern ist es nicht von Bedeutung, ob die Gelder dafür aus Gebühren oder Steuereinnahmen kommen: Es kann sogar argumentiert werden, dass Steuereinnahmen effizienter sind, da keine zusätzlichen Kosten der Erhebung anfallen. Von nicht-ökonomischer Seite wird die Erhebung über Steuern kritisiert, da erstens eine direkte politische Beeinflussung möglich würde und sie zweitens kein direktes Zurechnungssystem zwischen NutzerInnen und Anbietenden bedeute (was bei einer Dichte an Rundfunkgeräten in Haushalten von an die 100\% kaum mehr ins Gewicht fällt; auch Gebührenfinanzierung stellt nur eine Annäherung dar: wer konsumieren könnte, wird zur Gebührenabgabe verpflichtet, eine Kontrolle ist nur schwer möglich).

- Bei privatwirtschaftlicher Organisation von Rundfunkunternehmen hätte sich Fiskalpolitik als diskretionäre Förderung bewusst auf Maßnahmen zu konzentrieren, die Vielfalt fördern. ${ }^{20}$ Dabei muss aber in Rechnung gestellt werden, dass dies aufgrund der Kostensituation zu Lasten der technischen Effizienz des Sektors geschehen kann; dies gilt dann, wenn große Unternehmen kostengünstiger produzieren könnten als mehrere kleine.

Eine Diversifizierung der Inhalte von Massenmedien ist aber mit wirtschaftspolitischen Maßnahmen alleine nicht zu erreichen. Für das Beispiel des Fernsehsektors sind die Eigentumsrechte an bestehendem Programmmaterial privatwirtschaftlich hochkonzentriert; daher steigen mit zunehmender Nachfrage durch mehr TV-Stationen auch die Preise für Programme, die Finanzierungserfordernisse der einzelnen TV-Stationen erhöhen sich dementsprechend, während als Alternative nur die Verbilligung anderer Programmteile verbleibt.

Wenn Wirtschaftspolitik als oberstes Ziel „gesellschaftliche Wohlfahrt“ hat, dann reicht der Markt als Allokationsmechanismus nicht aus, jene Vielfalt der Medieninhalte zu garantieren, die Grundlage sowohl für eine funktionierende Demokratie als auch zur Zielerreichung der Wirtschaftspolitik notwendig ist, insbesondere auf der Ebene der inhaltlichen Gestaltung der Produkte. Meiner Einschätzung nach stellt das immer wieder propagierte Gegenkonzept der Zivilgesellschaft, auf so hochkomplexe Bereiche wie die Medienindustrie angewandt ${ }^{21}$, keinen praktikablen Lösungsansatz dar, sondern eine sowohl zeitliche wie auch inhaltliche Überforderung der diese Zivilgesellschaft tragenden citoyen. Wirtschaftspolitik als nur die Ökonomisierung befördernd abzuqualifizieren hieße andererseits, sich eines gesellschaftlichen Instrumentariums zu berauben, das nicht

19 Wie derzeit z. B. in den Niederlanden öffentlich-rechtlicher Rundfunk finanziert wird.

20 In Großbritannien oder Irland wird dies durch einen Anteil der Fremdproduktion von Fernsehstationen, der an unabhängige Produzenten vergeben werden muss, zu erreichen versucht. Dass dies weder zu verbesserten Arbeitsbedingungen, noch zu geringeren Abhängigkeiten zwischen Produzenten und Rundfunkstationen, noch zu diversifizierteren Inhalten geführt hat, zeigen Saundry/ Nolan (1998) für Großbritannien.

21 Wie auch gleichermaßen als Beispiel die Regulierung von Finanzmärkten angeführt werden könnte. 
nur durch ein anderes keineswegs adäquat ersetzt werden kann, sondern dessen differenzierter und kalkulierter Einsatz eine der wesentlichen Voraussetzungen für die Formation (und das Funktionieren) solcher Zivilgesellschaften bildet.

\section{Konklusio}

Die verstärkte Ausrichtung auf den Markt als bestes Allokationssystem, verbunden mit erhöhter Profitorientierung, Einfluss der Werbewirtschaft und dem Bemühen um Effizienzsteigerung wurde als Ökonomisierung bezeichnet. Dies wird nicht zuletzt durch die neoliberale Ausrichtung der Wirtschaftspolitik als Ermöglicher von Marktbedingungen erreicht. Somit reduziert sich Wirtschaftspolitik oftmals (auf die positiven Kräfte des Wettbewerbs vertrauend) darauf, ein reibungsloses Funktionieren des Marktes sicherzustellen. Noch stärker als in anderen Industrien ist am Massenmediensektor, aufgrund der Kostenstrukturen und spezifischen Gütereigenschaften (Kiefer 2001, Heinrich 1999), diese Wettbewerbsform allerdings ökonomisch nicht zu erreichen (bzw. sind Konzentrationen weitaus effizienter, gibt es hohe Marktmacht der Anbieterseite, ein Fehlen von Konsumentensouveränität und daher eine eingeschränkte Produktpalette). Damit ist die vorrangige Ausrichtung der Wirtschaftspolitik auf Wettbewerbspolitik wenig sinnvoll. Die Verschiebung der Eigentumsstrukturen im Rundfunk zugunsten privatwirtschaftlich geführter Unternehmen wie auch ein Abrücken der Wirtschaftspolitik von der Überzeugung, Massenmedienprodukte seien als öffentliche bzw. meritorische Güter zu behandeln, führt dazu, dass ein öffentliches Interesse an diesen Produkten von Seiten der Wirtschaftspolitik nur mehr nachrangig behandelt wird.

Wenn die „Ökonomisierung der Medien“ in ihrer Bedeutungszuschreibung aus obigen Gründen nicht in eindeutig positiver Konnotation verortet ist, so sind wirtschaftspolitische Maßnahmen notwendig und - auf die öffentliche Aufgabe und öffentliche Guteigenschaften fokussiert - auch von Nutzen. Wirtschaftspolitik wird gebraucht, um den Marktgesetzen - die durch Ökonomisierung stärker in die Medienindustrie eingreifen - andere ökonomische oder soziale Ziele entgegenzuhalten. Hier darf die Wirtschaftspolitik nicht aus ihrer Verantwortung entlassen werden.

Die neoklassische Theorie geht davon aus, dass der Markt, sich selbst überlassen, die beste Allokationsform der Ökonomie darstellt. Dies wird von sowohl keynesianischen Ansätzen wie auch der französischen Regulationstheorie widerlegt, vor allem aber von empirischen Befunden: Regulierungsaktivitäten (wie Wirtschaftpolitik) sind daher für das effiziente Funktionieren unserer kapitalistisch strukturierten Ökonomie notwendig.

Selbstredend ist Politik (und daher auch Wirtschaftspolitik) nicht unabhängig, sie braucht vielmehr wieder Kontrolle und klare (in unserem Falle: wirtschaftspolitische) Zielvorgaben, kann aber als Gegengewicht zum Markt fungieren und somit die negativen Auswirkungen des Marktes regeln. Dass die Politik wieder von Massenmedien abhängig ist, wie Medien von Profitinteressen abhängig sind, darf ebenso wenig außer Acht gelassen werden. Dabei ist zu berücksichtigen, dass Massenmedien sowohl Objekte als auch Träger von Wirtschaftspolitik ${ }^{22}$ sind; zweiteres, indem sie Präferenzen zwar keineswegs ausschließlich bilden, so doch über die zur Verfügung gestellten Informationen beeinflussen. Den Massenmedien kommt ihre ökonomische Bedeutung innerhalb der Gesamtwirtschaft nicht aufgrund ihrer herausragenden Wirtschaftskraft zu (gemessen

22 Ewald Nowotny $(1997,27 f$.) nennt Medien als Träger von Wirtschaftspolitik neben den politischen Parteien, Interessensgruppen und der staatlichen Bürokratie. 
am Anteil des Bruttoinlandsproduktes, der mit einigen wenigen Prozent für die gesamte Medienindustrie wenn schon nicht marginal, so doch wenig bedeutend ist), sondern vielmehr aufgrund ihres symbolischen Gehalts, ihrer Gestaltungskraft. Wirtschaftspolitik müsste den Spagat schaffen, auf Rahmenbedingungen zur Inhaltsproduktion regulierend einzugreifen, ohne inhaltlich zu regulieren. Wie dies ablauftechnisch zu bewerkstelligen wäre, ist damit noch keineswegs beantwortet. Ein intensiverer wissenschaftlicher Diskurs wäre allenfalls notwendig, um die Frage, wie dies gewährleistet werden könnte, hinlänglich beleuchten zu können. Die Schwierigkeit besteht darin, dass ökonomische Entwicklungen einer anderen Eigenlogik folgen und eine andere Dynamik nehmen, als gesellschaftspolitische Anforderungen an die Medienbranche dies tun. Hier die notwendigen Kongruenzen herzustellen, ist gleichermaßen eine Herausforderung für die Politik und für die Wissenschaft.

\section{Literatur}

Achille, Y./Miège, B. (1994): The limits to the adaptation strategies of European public service television. In: Media, Culture and Society, vol. 16, No. 1, S. 31 - 46.

Altmann, Jörn (2000): Wirtschaftspolitik, Stuttgart.

Altvater, Elmar (1997): Die Zukunft des Marktes, Münster.

Arestis, Philip/Sawyer, Malcolm (Hrsg.) (1998): The Political Economy of Economic Policies. Houndsmills u. a.

Bagdikian, Ben H. (1997): The Media Monopoly. 5. Aufl., Boston, Mass.

Berg, Hartmut/Cassel, Dieter (1995): Theorie der Wirtschaftspolitik; in: Bender u. a. (Hrsg.): Vahlens Kompendium der Wirtschaftstheorie und Politik, München, S. 163 - 238.

Blumler, Jay G. (Hrsg.) (1992): Television and the Public Interest. Vulnerable Values in West European Broadcasting, London/Newbury Park/New Delhi.

Boyer, Robert (1986): La théorie de la régulation: Une analyse critique, Paris.

Brown, Allan (1996): Economics, Public Service Broadcasting and Social Values. In: Media Economics 1/1996, S. 3 - 15.

Burgelmann, Jean Claude (1997): Issues and Assumptions in Communications Policy and Research in Western Europe: A critical Analysis. In: Corner, John/Schlesinger, Philip/Silverstone, Rodger (Hrsg.): International Media Research. A Critical Survey, London/New York, S. 123-153.

Coase, Ronald (1966): The Economics of Broadcasting and Government Policy. In: American Economic Review 2/1966, S. $440-447$.

Collins, Richard/Garnham, Nicholas/Locksley, Gareth (1988): The Economics of Television. The UK Case, London.

Götschl, Herbert (1998): Monopole fallen langsam. Vom Ende des ORF-Monopols und von der neuen Vielfalt am Hörfunksektor. In: Österreichische Zeitschrift für Politikwissenschaft 2, S. $117-128$.

Grisold, Andrea (1996): Regulierungsreformen am Mediensektor. Der „Fall“ Österreich, Frankfurt am Main/New York.

Grisold, Andrea (1998): Kopf oder Zahl? Konzentrationsprozesse und Regulierungsreformen am Mediensektor. In: Österreichische Zeitschrift für Politikwissenschaft 2, S. $129-142$.

KEG (2000): Merger Procedure Article 6(1)(b) Decision, Case No COMP/JV.37 - BSkyB/KirchPayTV, Luxembourg, 21.3.2000.

Heinrich, Jürgen (1999): Medienökonomie. Band 2: Hörfunk und Fernsehen, Opladen.

Herman, Edward/Mc Chesney, Robert (1997): The Global Media. The New Missionaries of Corporate Capitalism, London/Washington.

Kiefer, Marie-Luise (1997): Privatisierung - cui bono? In: Medienjournal 2/1997, S. 4 - 13.

Kiefer, Marie-Luise (2001): Medienökonomik, München.

McQuail, Denis (1998): Commercialization and Beyond. In: McQuail, Denis/Siune, Karen (Hrsg.): Media Policy. Convergence, Concentration and Commerce, London u. a., S. 107 - 127.

Nowotny, Ewald (1997): Grundlagen und Institutionen der Wirtschaftspolitik. In: Nowotny, 
Ewald/Winckler, Georg (Hrsg.): Grundzüge der Wirtschaftspolitik Österreichs, Wien, S. 11 48.

Peltzman, Sam (1976): Toward a more General Theory of Regulation. In: Journal of Law and Economics, 19 , S. $211-240$.

Peterson, Richard A. (1982): Five Constraints on the production of Culture: Law, Technology, Market, Organizational Structure and Occupational Careers. In: Journal of Popular Culture, 16, S. $142-153$.

Pirker, Reinhard (1999): Die Ökonomisierung des öffentlichen Diskurses oder: Woher kommt die neoliberale Rhetorik? In: Schmee, Josef/Weissel, Erwin (Hrsg.): Die Armut des Habens, Wien, $27-37$.

Rothschild, Kurt (1996): Bemerkungen zur Ökonomie, Politik und Ethik des Wohlfahrtsstaates. In: Kurswechsel 3/1996, S. $37-60$.

Saundry, Richard/Nolan, Peter (1998): Regulatory change and performance in TV production, in: Media, Culture and Society 2/1998, S. $409-426$.

Stigler, George (1971): The Theory of Economic Regulation. In: Bell Journal of Economics, Vol. 2, S. $3-21$.

Stigler, George (Hg. 1995): Chicago studies in political economy, Chicago. 